\title{
Multi-hop Communication between LoRa End Devices
}

\author{
Yosefine Triwidyastuti ${ }^{1}$, Musayyanah ${ }^{2}$, Fifin Ernawati ${ }^{3}$, Charisma Dimas \\ Affandi ${ }^{4}$ \\ ${ }^{1,2,3,4}$ Computer Engineering Study Program, Faculty of Technology and Informatics, \\ Universitas Dinamika, Indonesia \\ Email: 'yosefine@dinamika.ac.id, ${ }^{2}$ musayyanah@dinamika.ac.id
}

\begin{abstract}
Gateway elimination in a LoRa network could highly reduce the network installation cost. However, LoRa end devices could not overcome many obstacles with only a point-to-point communication. Thus, this research implemented a multi-hop communication in a LoRa network. One or more LoRa end devices are placed between the source node and the destination node to act as relay nodes. A simple routing based on the packet length is configured to determine the packet transmission path. As the results, the designed multi-hop communication could improve packet success rate until 2,47 times in indoor environment. Whereas, the optimum delay time for multi-hop communication is $100 \mathrm{~ms}$ for each hop to produce high PRR and lowest RTT.
\end{abstract}

Keywords: LoRa, End Device, Multi-hop, Relay Node

\section{INTRODUCTION}

Wireless Communication Systems have been widely applied in various fields, such as environment [1-2] and health monitoring [3-4] in real time. The desired communication system is communication with low power, high range, low-cost, and reliable using LPWAN (Low-Power Wide Area Network) technology. Much research has developed reliable communication with low power, as in [3-5]. One example of new emerging LPWAN is LoRa (Long Range). LoRa is a low-priced module that can send information with a maximum distance of $15 \mathrm{~km}$.

LoRa performance has been analyzed in rural, suburban and urban areas [6]. According to Ramon, LoRa's performance depends on the conditions of the propagation environment scenario [7]. In an indoor environment with significant obstacles such as walls, the maximum distance is 45 meters [8].

The Wireless Sensor Network (WSN) has also been extensively researched because this technology includes several nodes that can be deployed in obstacle environments. In order to reduce packet losses, a modified communication system is needed that can forward information to the receiver. The communication system is referred to as a multi-hop communication system. In a multi-hop communication system, a packet must be delivered through several paths to reach its destination, and communication between two nodes is delivered by an intermediate node. The benefit of multi-hop is overcoming power reduction [9].

A multi-hop communication system that uses Zigbee and Sigfox has been implemented [10], where the communication system is carried out in an indoor environment with a 
low range. This communication system consists of a source node (transmitter), a relay node, and a destination node (receiver).

There has also been a research concerned on implementing a Mesh network using Lora [2]. However, the network used many LoRa gateways that have expensive price. LoRa gateway costs until 3,5 million rupiahs, while LoRa end device only costs 150 thousand rupiahs [11]. A LoRa network that only consists of end devices would be very advantageous, because it could highly reduce the installation cost.

The objective of this research is to build a multi-hop communication using LoRa network that consists of several end devices without any gateway. One or two LoRa end devices are placed between the transmitter and receiver to act as repeaters. This research also studied the performance difference for various hop number, delay time, and packet length. Moreover, the experiments were conducted in indoor and outdoor environment.

\section{METHODS}

The block diagram of multi-hop communication used in this research is shown in Figure 1. The communication between the source node and destination node is relayed by one or two repeater nodes. After the packet is arrived at the destination node, a feedback packet is relayed again to the source node via repeater nodes.

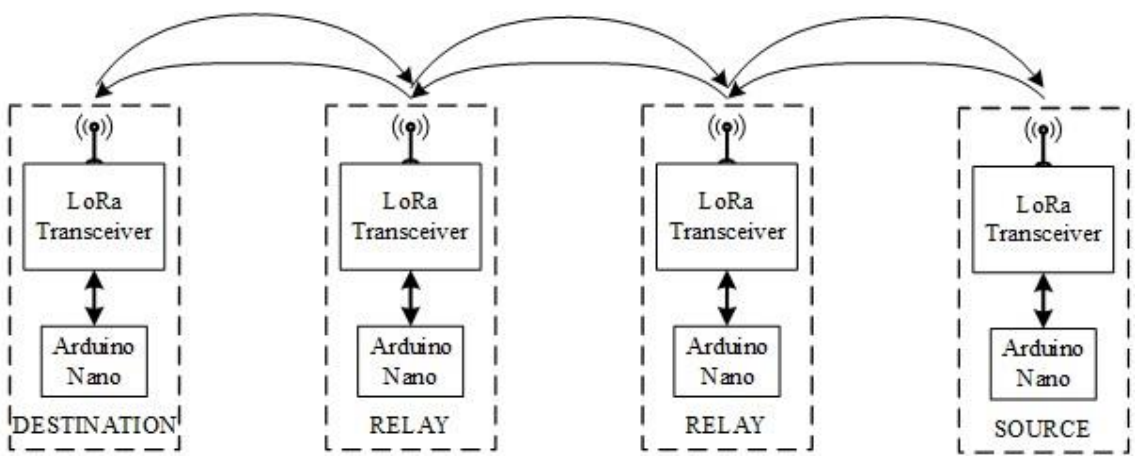

Figure 1. Block diagram of multi-hop communication

Each node in multi-hop communication contains of an Arduino Nano as the controller and a LoRa transceiver as the communication module. The module used in this research is HopeRF-RFM9x LoRa Module which has frequency range from $900 \mathrm{MHz}$ to 930 $\mathrm{MHz}$ that is suitable with LPWAN regulation in Indonesia [12]. This research used a default helical antenna which is available in the LoRa module.

Because the LoRa end device is a wireless transceiver module, every LoRa end device can receive other LoRa end devices' packets and transmit packets to other LoRa end devices within its coverage. In this research, the multi-hop communication between LoRa end devices is arranged by the packet format. Every LoRa end device should check its received packets to determine whether it should relay the packet or not. 
The different packet format for every hop is defined in Table 1. First, packets are sent from the source node with header " $T$ ". Then the neighbor nodes will add a trailer at the end of the packet before send it to other nodes. The format of the trailer is one character that defines the node ID, which are $\mathrm{T}$ for the source node, $\mathrm{R}$ for the destination node, $\mathrm{Y}$ for the first relay, and X for the second relay. Finally, every hop that has been passed by a packet makes the packet length becomes longer one byte.

Table 1. Packet format

\begin{tabular}{cccc}
\hline Type & Sent Node & Received Node & Format \\
\hline 1 & Source & Relay 1 & $\mathrm{~T}+$ time \\
2 & Relay 1 & Relay 2 & $\mathrm{~T}+$ time $+\mathrm{Y}$ \\
3 & Relay 2 & Destination & $\mathrm{T}+$ time + YX \\
4 & Destination & Relay 2 & $\mathrm{~T}+$ time + YXR \\
5 & Relay 2 & Relay 1 & $\mathrm{~T}+$ time + YXRX \\
6 & Relay 1 & Source & $\mathrm{T}+$ time + YXRXY \\
\hline
\end{tabular}

The addition in the packet length could simplify the routing process in multi-hop communication. Packet type 1 with no identification character in the trailer that makes it the shortest packet in the network is received only by the first relay node. On the other hand, packet type 6 that is the longest packet should be received only by source node.

The flowchart of the source node is shown in Figure 2. First, the transmitter node should build a packet contains of character "T" and the recorded time. Then the node transmits the packet and counts a timer of $2000 \mathrm{~ms}$ or two seconds. While in the waiting process, the transmitter node should always check its buffer to receive a returned packet. If the transmitter receives a packet during the waiting time, then the node should check the packet length whether it is the correct returned packet or not. If the returned packet is the correct packet, then the source node back to the process of transmitting next packet again. 


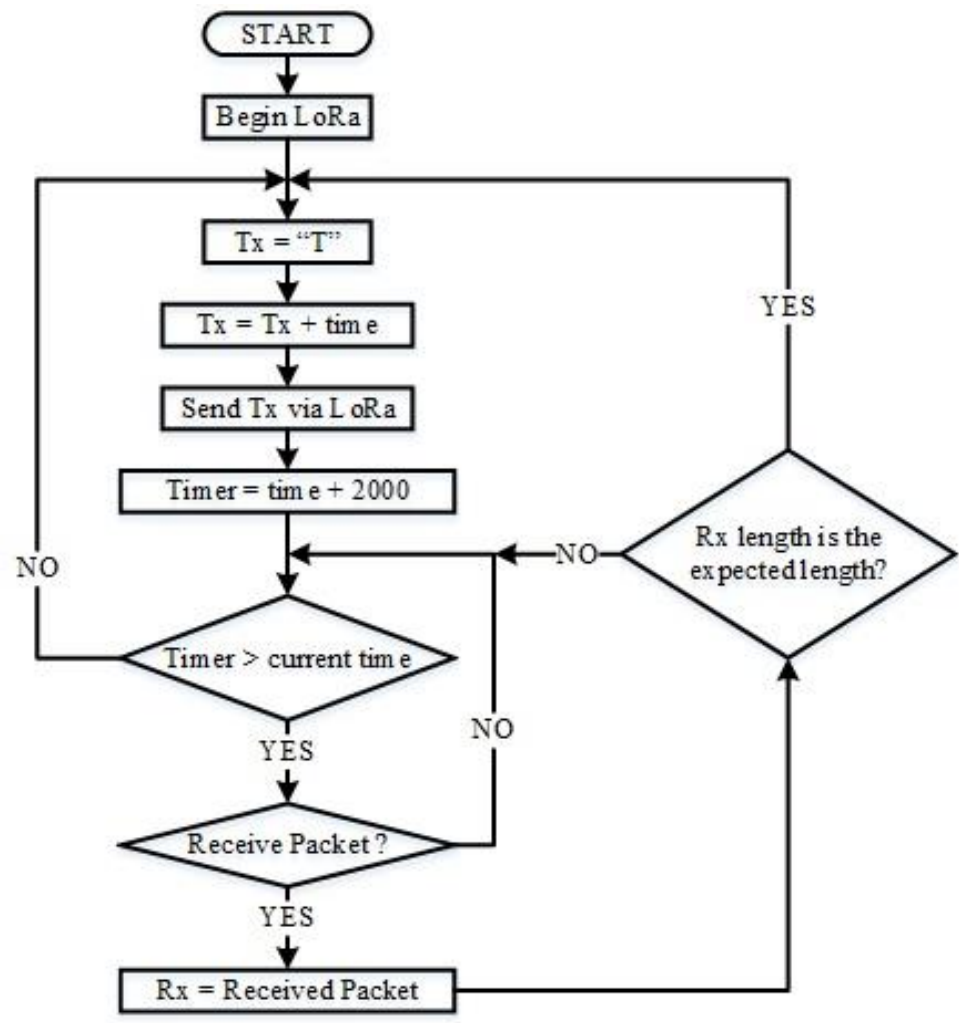

Figure 2. Source node flowchart

The flowchart of the relay nodes or the destination node is shown in Figure 3. Destination node uses the same process as in relay nodes, because all packets received by destination node should be transmitted back to the source node. This round-trip transmission could ensure the rate of the packet transmission has the same pace with the rate of the packet reception. 


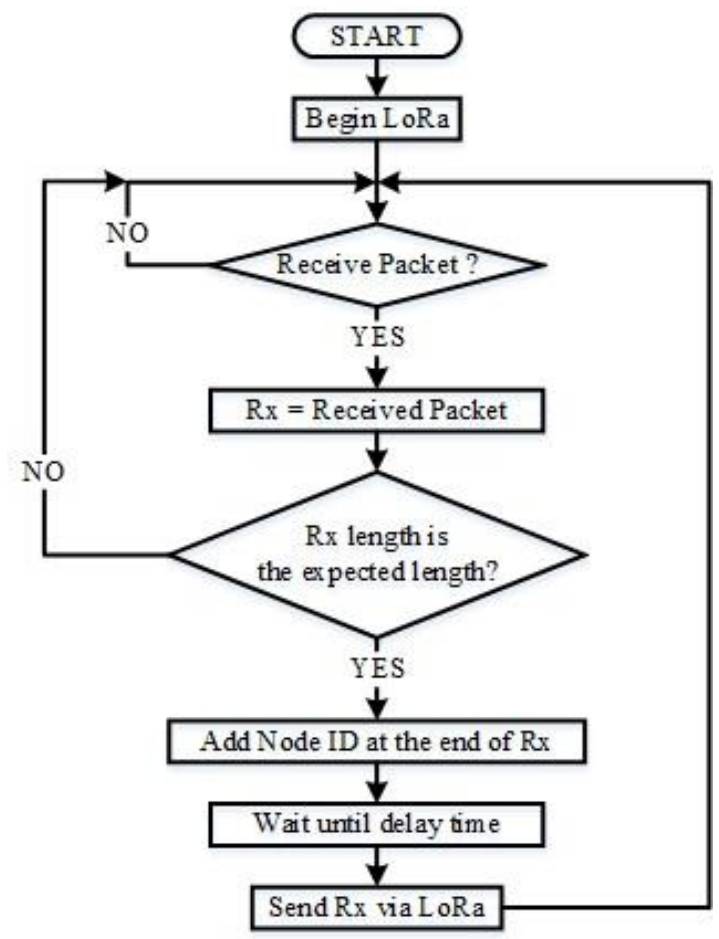

Figure 3. Relay node flowchart

The process in the repeater nodes and destination node begin with checking the LoRa's buffer to receive packets. When the node receives a packet, it checks the packet length based on the correct packet format. If the packet length is the correct expected length, then the node adds one character behind the packet string that defines its node identification character.

After adding the node ID, the relay node should wait in a certain time to avoid packet collision. Wireless communication using LoRa end devices is susceptible to packet collision because every node has ability to transmit at any time and no special device to control the transmission. Therefore, this research implemented a delay time to avoid any concurrent transmission. After the delay time runs out, the node should transmit the modified packet directly and then wait for the next received packet.

The performance of the multi-hop communication is calculated from the Packet Reception Ratio (PRR). The PRR value is the ratio between the number of received packet in source node $\left(N_{R}\right)$ and the number of sent packet from the source node $\left(N_{S}\right)$, as shown in (1). The received packet that is calculated in the PRR measurement should be pre-concerted that the packet has the same content with the transmitted packet.

$$
P R R=\frac{N_{R}}{N_{S}} \times 100 \%
$$


Other metric used to analyze the communication performance is the Round-Trip Time (RTT). The RTT value is calculated from the average of the recorded time each packet is received in the source node $\left(T_{R}\right)$ diminished by the recorded time that packet is transmitted by the source node $\left(T_{S}\right)$, as shown in (2). The received packet that is included in this time calculation is also should be pre-concerted that the packet has no error from the transmitted packet.

$$
R T T=T_{R}-T_{S}
$$

\section{RESULT AND DISCUSSION}

This research tested the multi-hop communication in several conditions, which are in indoor environment and outdoor environment. The research also tested different number of hops, from one hop or a point-to-point communication to three hop communication that yields a six hop round trip communication. Each experiment was conducted in a fixed duration of fifteen minutes.

The sketch for indoor test environment is shown in Figure 4. The first experiment tested the multi-hop communication between LoRa end devices that are placed in different rooms in the same floor. The source node was placed in Room 1 and destination node was placed in Room 4. The elevators, stairs, rest rooms, and other rooms between the source and destination node portrayed considerable obstacles for the wireless communication. The distance between Room 1 and Room 4 is 60 meters.

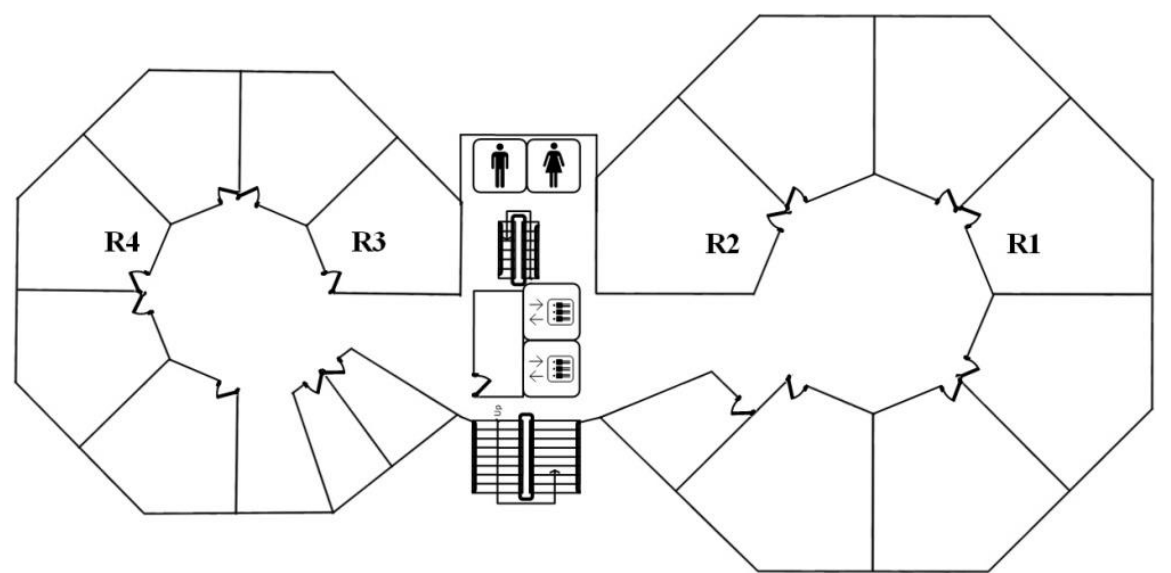

Figure 4. Floor map for indoor test

The first experiment was conducted in several hop numbers. When there is no relay node between source and destination node, the transmission is considered as a one hop communication. Two hop communications were tested by adding one relay node in Room 2 with several payload lengths and different delay configuration. Last, in the first experiment one relay node was added again and placed in Room 3 that makes it a threehop communication. 
The results of the first experiment that tested the indoor performance in the same floor are shown in Table 2. The payload lengths were varied between 5, 20 and 40 bytes. Besides, the delay time was also varied from $50 \mathrm{~ms}$ to $300 \mathrm{~ms}$. The highest PRR is obtained from the transmission of 5-byte packet in two hop communication using delay time of $200 \mathrm{~ms}$ and $300 \mathrm{~ms}$. However, the transmission yielded a high RTT.

Table 2. Indoor test results

\begin{tabular}{ccccc}
\hline Hop Count & Payload & Delay & PRR & RTT \\
\hline 1 & 5 bytes & $50 \mathrm{~ms}$ & $76.19 \%$ & $85.13 \mathrm{~ms}$ \\
2 & 5 bytes & $50 \mathrm{~ms}$ & $98.14 \%$ & $257.86 \mathrm{~ms}$ \\
2 & 5 bytes & $100 \mathrm{~ms}$ & $99.05 \%$ & $408.13 \mathrm{~ms}$ \\
2 & 5 bytes & $200 \mathrm{~ms}$ & $99.08 \%$ & $707.29 \mathrm{~ms}$ \\
2 & 5 bytes & $300 \mathrm{~ms}$ & $99.08 \%$ & $1006.91 \mathrm{~ms}$ \\
2 & 20 bytes & $50 \mathrm{~ms}$ & $97.40 \%$ & $352.27 \mathrm{~ms}$ \\
2 & 20 bytes & $100 \mathrm{~ms}$ & $98.29 \%$ & $502.15 \mathrm{~ms}$ \\
2 & 40 bytes & $50 \mathrm{~ms}$ & $96.81 \%$ & $455.93 \mathrm{~ms}$ \\
2 & 40 bytes & $100 \mathrm{~ms}$ & $97.41 \%$ & $606.15 \mathrm{~ms}$ \\
3 & 5 bytes & $50 \mathrm{~ms}$ & $97.45 \%$ & $436.90 \mathrm{~ms}$ \\
3 & 5 bytes & $100 \mathrm{~ms}$ & $98.02 \%$ & $686.54 \mathrm{~ms}$ \\
3 & 20 bytes & $100 \mathrm{~ms}$ & $97.40 \%$ & $846.00 \mathrm{~ms}$ \\
3 & 40 bytes & $100 \mathrm{~ms}$ & $96.63 \%$ & $1016.96 \mathrm{~ms}$ \\
\hline
\end{tabular}

The comparison of different delay time configuration is shown in Figure 5. The experiment used payload length of 5 bytes and two-hop communication. Longer delay produces higher PRR and RTT. From the comparison, the optimum delay time is 100 ms which produces the same high PRR with other delay time but results the lowest RTT.

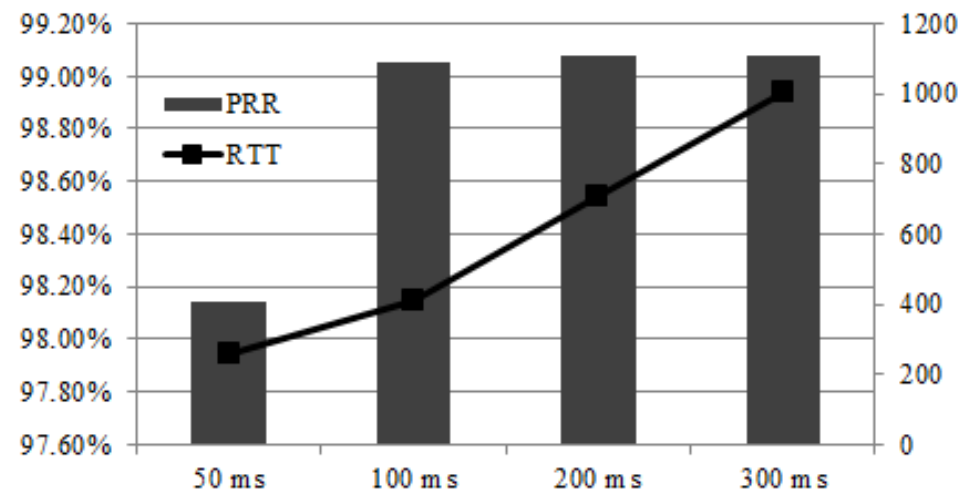

Figure 5. Delay time comparison

The comparison of different hop number in multi-hop communication is shown in Figure 6 . The experiment used payload length of 5 bytes and delay time of $50 \mathrm{~ms}$. From the comparison, three hop PRR is lower than two-hop. This indicates that more hops could not ensure good PRR because more hop means more nodes and more traffic. The 
arrangement of adequate hop should be considered based on the node coverage. Then in next experiment, this research only used two hops.

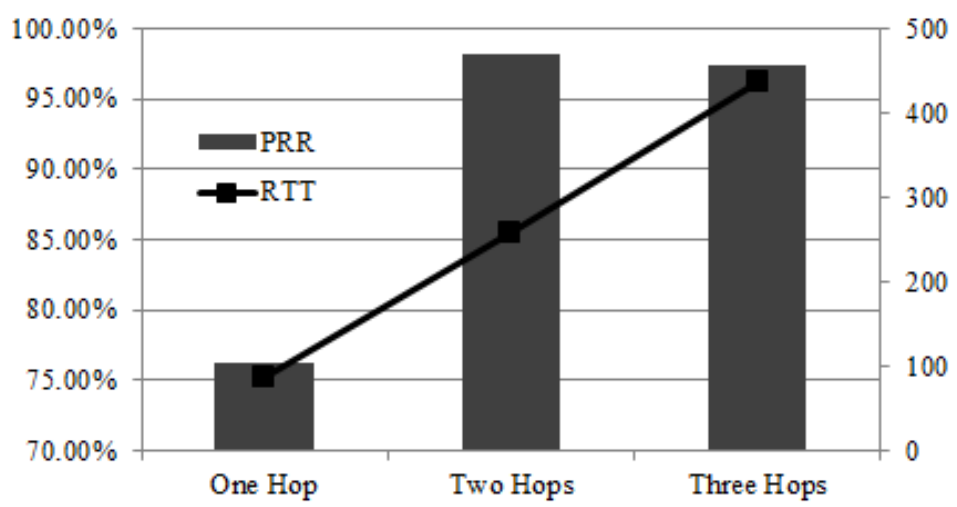

Figure 6. Hop count comparison

The effect of different packet length in two hop communication is shown in Figure 7. The experiment also analyzed two delay variations, which are $50 \mathrm{~ms}$ and $100 \mathrm{~ms}$. Longer packet resulted in lower PRR and higher RTT. This 1\% PRR decrement happened in two delay configurations. However, $50 \mathrm{~ms}$ delay has lower PRR than 100 ms delay, because $50 \mathrm{~ms}$ delay is too short for multi nodes to avoid packet collision in a wireless network.

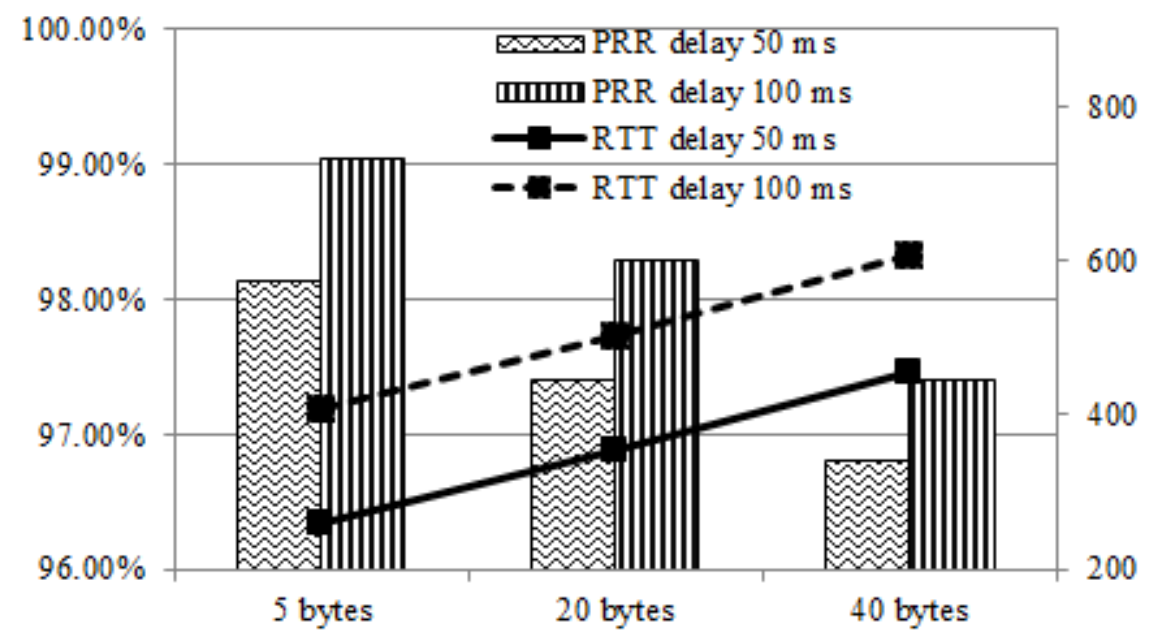

Figure 7. Comparison of different packet length

The second experiment was conducted in the same indoor environment but in different floors that makes the obstacles more impenetrable. The source node was placed in Room 1 of the fifth floor, while the destination node was placed in Room 4 of the third 
floor. Two-hop communication was tested by added one relay node in Room 3 of the fifth floor. The results of the second experiment are shown in Table 3.

Table 3. Test result for different floor

\begin{tabular}{ccccc}
\hline Hop Count & Payload & Delay & PRR & RTT \\
\hline 1 & 5 bytes & $50 \mathrm{~ms}$ & $39.36 \%$ & $85.12 \mathrm{~ms}$ \\
2 & 5 bytes & $50 \mathrm{~ms}$ & $97.24 \%$ & $258.17 \mathrm{~ms}$ \\
2 & 5 bytes & $100 \mathrm{~ms}$ & $99.70 \%$ & $408.11 \mathrm{~ms}$ \\
2 & 20 bytes & $50 \mathrm{~ms}$ & $96.12 \%$ & $352.06 \mathrm{~ms}$ \\
2 & 20 bytes & $100 \mathrm{~ms}$ & $97.20 \%$ & $502.15 \mathrm{~ms}$ \\
2 & 40 bytes & $50 \mathrm{~ms}$ & $95.77 \%$ & $456.63 \mathrm{~ms}$ \\
2 & 40 bytes & $100 \mathrm{~ms}$ & $98.80 \%$ & $606.31 \mathrm{~ms}$ \\
\hline
\end{tabular}

The performance improvement using multi-hop communication can be seen from the higher PRR in two hop communication than in one hop communication. The PRR value is increased from $39.36 \%$ to $97.24 \%$ for 5-byte packet transmission using 50 ms delay. This means the performance is improved until 2,47 times, even though the RTT is also increased until around $173 \mathrm{~ms}$.

Other test was also conducted in outdoor environment, which is in housing. The sketch for outdoor test environment is shown in Figure 8. The source node and the destination node are separated by seven houses that can be considered as significant obstacles. In order to test the two-hop communication, one relay node is placed in the middle of the source and destination node.

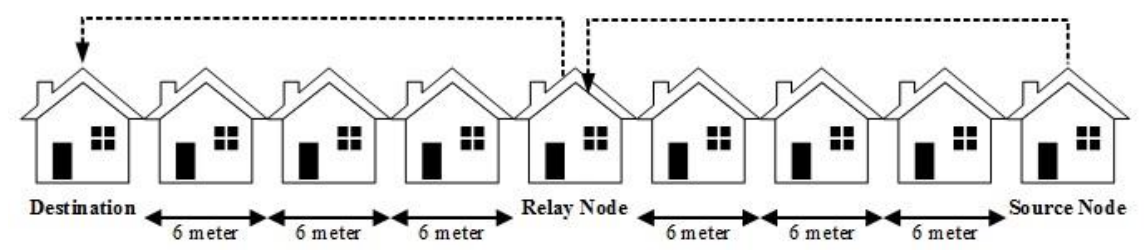

Figure 8. Housing map for outdoor test

The result of the outdoor test is shown in Table 4. Significant performance of two hop communication could increase the PRR value from $8.11 \%$ to $96.63 \%$ or around 12 times for a 5-byte packet transmission with $50 \mathrm{~ms}$ delay. This good performance of two hop communication could cover far node with the help of a relay node. Although the RTT is also increased, the low RTT increment of only 173 ms could be compensated by the less packet errors. Good achievement in these multi-hop experiments between LoRa end devices could reduce the gateway installation in other experiment, such as [2].

Table 4. Housing test result

\begin{tabular}{ccccc}
\hline Hop Count & Payload & Delay & PRR & RTT \\
\hline 1 & 5 bytes & $50 \mathrm{~ms}$ & $8.11 \%$ & $85.03 \mathrm{~ms}$ \\
2 & 5 bytes & $50 \mathrm{~ms}$ & $96.63 \%$ & $258.33 \mathrm{~ms}$ \\
2 & 5 bytes & $100 \mathrm{~ms}$ & $99.06 \%$ & $408.28 \mathrm{~ms}$
\end{tabular}




\begin{tabular}{ccccc}
2 & 20 bytes & $50 \mathrm{~ms}$ & $87.70 \%$ & $352.39 \mathrm{~ms}$ \\
2 & 20 bytes & $100 \mathrm{~ms}$ & $97.12 \%$ & $502.32 \mathrm{~ms}$ \\
2 & 40 bytes & $50 \mathrm{~ms}$ & $86.17 \%$ & $458.18 \mathrm{~ms}$ \\
2 & 40 bytes & $100 \mathrm{~ms}$ & $95.58 \%$ & $606.79 \mathrm{~ms}$ \\
\hline
\end{tabular}

\section{CONCLUSION}

The multi-hop communication could improve the packet transmission for until 2.47 times in indoor environment. However, too many hops could decrease the communication performance because there would be more traffic from many nodes. The optimum delay configuration for multi-hop communication is $100 \mathrm{~ms}$ that could result in high PRR and lowest RTT.

\section{ACKNOWLEDGMENT}

Authors would like to thank the Ministry of Research, Technology and Higher Education for the research grant based on the Research Contract Fiscal Year 2019 number 113/SP2H/LT/DRPM/2019.

\section{REFERENCES}

[1] Hulu, E., Riyanto, B., \& Widyantoro, S. (2015). Wireless Sensor Networks for Volcano Activity Monitoring: A Survey. Scientific Journal of Informatics, 2(1), 53-62.

[2] Ebi, C., Schaltegger, F., Rust, A., \& Blumensaat, F. (2019). Synchronous LoRa Mesh Network to Monitor Processes in Underground Infrastructure. IEEE Access, 7, 57663-57677.

[3] Catherwood, P. A., Steele, D., Little, M., McComb, S., \& McLaughlin, J. (2018). A Community-Based IoT Personalized Wireless Healthcare Solution Trial. IEEE Journal of Translational Engineering in Health and Medicine, 6, 1-13.

[4] Wu, F., Redoute, J-M, \& Yuce, M. R. (2018). WE-Safe: A Self-Powered Wearable IoT Sensor Network for Safety Applications Based on LoRa. IEEE Access, 6, 40846-40853.

[5] Tome, M. C., Nardelli, P. H. J. \& Alves, H. (2019). Long-range Low-power Wireless Networks and Sampling Strategies in Electricity Metering. IEEE Transactions on Industrial Electronics, 66(2), 1629-1637.

[6] Chall, R. E., Lahoud, S. \& Helou, M. E. (2019). LoRaWAN Network: Radio Propagation Models and Performance Evaluation in Various Environments in Lebanon. IEEE Internet of Things Journal, 6(2), 2366-2378.

[7] Sanchez-Iborra, R., Sanchez-Gomez, J., Ballesta-Viñas, J., Cano, M-D, \& Skarmeta, A. F. (2018). Performance Evaluation of LoRa Considering Scenario Conditions. Sensors, 18(3), 772.

[8] Triwidyastuti, Y. (2019). Performance Analysis of Point-to-Point LoRa End Device Communication. Lontar Komputer, 10(3), 140-149.

[9] Babun, L., Yurekli, A. I., \& Guvenc, I. (2015). Multi-hop and D2D Communications for Extending Coverage in Public Safety Scenarios. 40 ${ }^{\text {th }}$ Annual IEEE Conference on Local Computer Networks, Florida: 26-29 October.

[10] Sarijari, M. A. B., Rashid, R. A., Rahim, M. R. A., \& Mahalin, N. H. (2008). Wireless Home Security and Automation System Utilizing ZigBee based Multihop Communication. $6^{\text {th }}$ National Conference on Telecommunication 
Technologies and $2^{\text {nd }}$ Malaysia Conference on Photonics, Malaysia: 26-28 August.

[11] Ciuffoletti, A. (2018). Low-Cost IoT: A Holistic Approach. Journal of Sensor and Actuator Networks, 7(2), 19.

[12] Ismail. (16 May 2019). Persyaratan Teknis Alat dan/atau Perangkat Telekomunikasi Low Power Wide Area. Regulation of the General Director of Resources and Devices of Post and Informatics. Jakarta: Ministry of Communication and Informatics. 